\title{
Prevalence and characteristics of Kawasaki disease before and during the COVID-19 pandemic
}

\author{
Rintaro Ono ${ }^{1}\left[\right.$ Daisuke Hasegawa $^{1}$
}

Received: 22 October 2020 / Accepted: 3 January 2021 / Published online: 6 February 2021

(c) Children's Hospital, Zhejiang University School of Medicine 2021

Kawasaki disease $(\mathrm{KD})$ is a febrile disease with unclear pathogenesis. Thus far, KD has been considered to be triggered, at least in part, by an infection [1]. In 2020, coronavirus disease 2019 (COVID-19) became a global pandemic and lifestyles changed drastically. Consequently, the prevalence of common infection has decreased significantly. This epidemiological change prompted us to postulate that major variations of common pediatric infections would affect the incidence of KD.

We retrospectively analyzed 447 patients with KD, including patients with incomplete $\mathrm{KD}$, treated at our hospital between January 2012 and August 2020. KD patients diagnosed after March 2020 were defined as the "during COVID-19 pandemic" group, whereas those diagnosed prior to December 2019 were defined as the "before COVID-19 pandemic" group. A diagnosis of KD was assigned based on the previous report [2], and treatment was provided based on the RAISE study [3]. To maintain infection control of the pediatric ward, patients with respiratory symptoms were screened for respiratory syncytial virus and human metapneumovirus, and patients with pharyngeal findings were screened for streptococci or adenovirus, by rapid antigen tests. During the influenza epidemic period, all patients were tested for rapid antigen test of influenza. Six patients diagnosed with KD between January 2020 and February 2020, and 37 patients ( 2 patients in "during COVID-19 pandemic group", and 35 patients in "before COVID-19 pandemic group") treated differently from the RAISE study (i.e., spontaneous remission without IVIG administration within 4 days), were excluded from the current analysis. PCR testing for SARS-CoV-2 was done as part of the clinical examination at a commercial laboratory. Patients' plasma or serum collected before KD treatment was cryopreserved

Rintaro Ono

rin.r34@gmail.com

1 Department of Pediatrics, St. Luke's International Hospital, 9-1 Akashicho, Chuo-ku, Tokyo 104-8560, Japan after obtaining written informed consent for the research use from the guardians. Antibodies for SARS-CoV-2 were evaluated using SARS-CoV-2 IgM/IgG (Artron Laboratories Inc. Canada). The sensitivity and specificity of this kit are $98 \%$ and $95 \%$, respectively, when analyzed more than 2 weeks after SARS-CoV-2 infection. For a statistical analysis, the monthly incidences of each disease "before" pandemic (2015-2019) and "during" pandemic (2020) were compared using Wilcoxon signed-rank sum test. Our study was approved by the Institutional Review Board at St Luke's International hospital (18-R103, 19-J016).

Twenty patients were diagnosed with KD during the COVID-19 pandemic, and 384 were diagnosed between January 2012 and December 2019. The prevalence of KD was comparable between the "before" and "during" COVID19 pandemic groups (Table 1). Table 1 illustrates the characteristics of the KD patients. Both groups had similar clinical manifestations, risk scores, and treatment response. No patients of "during" COVID-19 pandemic group exhibited positivity for PCR or antibody testing for SARS-CoV-2. No patients developed toxic shock syndrome or KD-like symptoms, which have been reported as multisystem inflammatory syndrome in children (MIS-C) after SARS-CoV-2 infection in the Western countries, whereas one patient developed KD-shock syndrome prior to the COVID-19 pandemic [4]. MIS-C may be a disease with racial differences because there are very few reports of MIS-C from East Asian countries, such as China and Japan [5, 6]. Another reason that we did not observe patients with MIS-C may be explained by the incidence of COVID-19 in children which was lower in Japan compared with Western countries during the research period.

Data from the National Institute of Infectious Diseases of Japan [7] revealed that the occurrence of most of the common infectious diseases among children, such as chickenpox, mumps, infectious gastroenteritis, and group A streptococcal pharyngitis, declined significantly using Wilcoxon signedrank sum test during the COVID-19 pandemic, whereas the incidence of less epidemic diseases, such as bacterial 
Table 1 Patients' characteristics

\begin{tabular}{lll}
\hline Variables & $\begin{array}{l}\text { Before COVID19 pandemic } \\
(N=384)\end{array}$ & $\begin{array}{l}\text { During COVID19 } \\
\text { pandemic }(N=20)\end{array}$ \\
\hline Age (mon) & $23(2-133)$ & $17(7-92)$ \\
Sex (M/F) & $234 / 150$ & $9 / 11$ \\
Kobayashi score & $3(0-10)$ & $4(0-7)$ \\
Complete KD & $291 / 384(76 \%)$ & $14 / 20(70 \%)$ \\
Principal symptoms & & \\
Fever & $383 / 384(99.7 \%)$ & $20 / 20(100 \%)$ \\
Bilateral conjunctival congestion & $347 / 384(90 \%)$ & $16 / 20(80 \%)$ \\
Changes of lips and oral cavity & $306 / 384(80 \%)$ & $16 / 20(80 \%)$ \\
Polymorphous exanthema & $323 / 384(84 \%)$ & $17 / 20(85 \%)$ \\
Changes of peripheral extremities & $286 / 384(74 \%)$ & $14 / 20(70 \%)$ \\
Non-purulent cervical lymphadenopathy & $269 / 384(70 \%)$ & $15 / 20(75 \%)$ \\
Additional treatment required & $65 / 384(17 \%)$ & $3 / 20(15 \%)$ \\
Coronary artery abnormality & $30 / 384(8 \%)$ & $1 / 20(5 \%)$ \\
Positive SARS-CoV-2 PCR & & $0 / 11(0 \%)$ \\
Positive SARS-CoV-2-IgG & & $0 / 9(0 \%)$ \\
Positive SARS-CoV-2-IgM & & $0 / 9(0 \%)$ \\
\hline
\end{tabular}

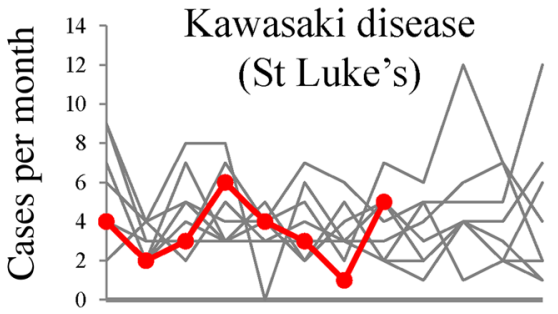

Exanthema subitum
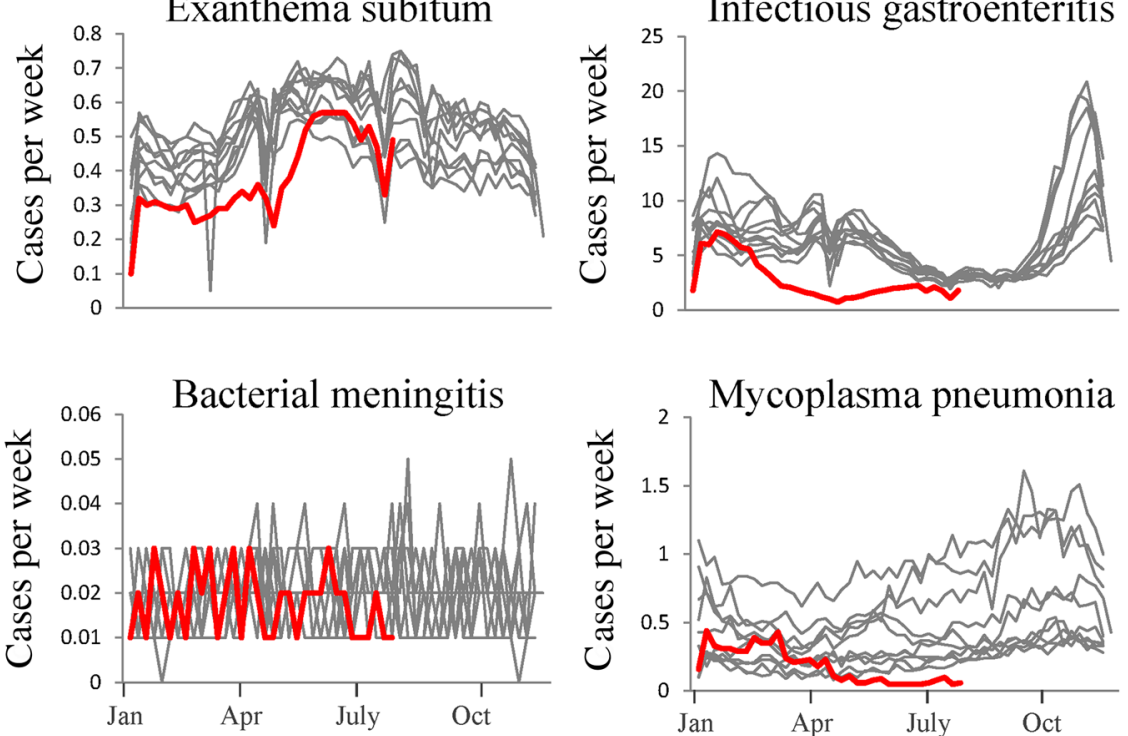

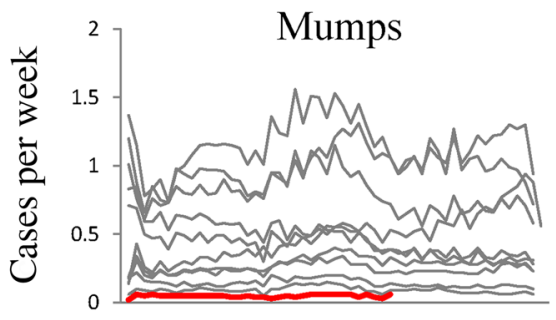

Herpangina

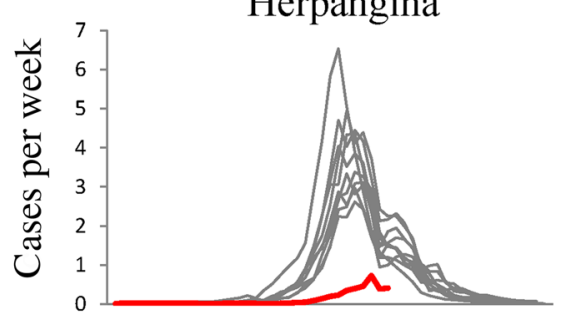

Group A

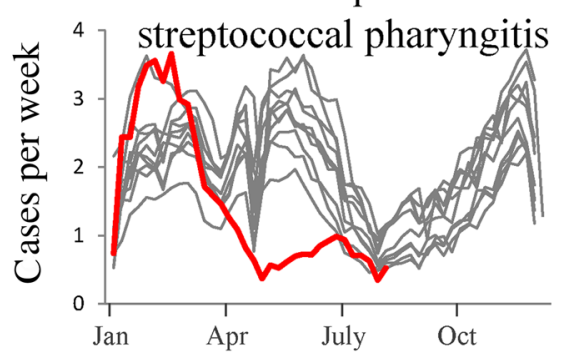

Fig. 1 Prevalence of Kawasaki disease and other infectious diseases in children. The prevalence of Kawasaki disease diagnosed at St Luke's International hospital between January 2012 and August 2020 is shown in the upper left panel. Weekly number of cases per sentinel of the week of other infectious diseases including chickenpox, mumps, exanthema subitum, infectious gastroenteritis, herpangina, bacterial meningitis, mycoplasma pneumonia, and group A streptococcal pharyngitis in Japan are also shown. These data are from National Institute of Infectious Diseases of Japan (https://www.niid. go.jp/niid/ja/data.html). Data on 2020 are indicated as a red line 
meningitis and exanthema subitum, did not change significantly (Fig. 1). It is noteworthy that the incidence of KD did not change during the COVID-19 pandemic. Although the number of cases was limited, our study suggested that incidence of KD might not be influenced by prevalence of contagious viral infection. Further study will be required to clarify the association between KD and infections.

Acknowledgements We would like to thank Dr. Osamu Takahashi, Dr. Hiroki Yoshihara, Dr. Yuki Uehara, St Luke's International Hospital, and Ms. Shizuka Yamagata-Uyama, St Luke's International University, for their help with this study.

Author contributions RO and DH designed the study, wrote the manuscript, and treated the patients. RO performed experiments. Both authors listed have made a substantial, direct and intellectual contribution to the work, and approved it for publication.

Funding This work was supported by JSPS KAKENHI Grant Number JP: 20K16937.

\section{Compliance with ethical standards}

Ethical approval This study was reviewed and approved by the Institutional Review Board at St Luke's International hospital (18-R103, 19-J016).

Conflict of interest No financial or nonfinancial benefits have been received or will be received from any party related directly or indirectly to the subject of this article.
Informed consent Written informed consent was obtained from parents when laboratory testing for research purposes is done.

\section{References}

1. Rowley AH, Wylie KM, Kim KY, Pink AJ, Yang A, Reindel R, et al. The transcriptional profile of coronary arteritis in Kawasaki disease. BMC Genomics. 2015;16:1076.

2. Ayusawa M, Sonobe T, Uemura S, Ogawa S, Nakamura Y, Kiyosawa N, et al. Revision of diagnostic guidelines for Kawasaki disease (the 5 th revised edition). Pediatr Int. 2005;47:232-4.

3. Kobayashi T, Saji T, Otani T, Takeuchi K, Nakamura T, Arakawa $\mathrm{H}$, et al. Efficacy of immunoglobulin plus prednisolone for prevention of coronary artery abnormalities in severe Kawasaki disease (RAISE study): a randomised, open-label, blinded-endpoints trial. Lancet. 2012;379:1613-20.

4. Ono R, Shimizu M, Yamamoto K, Umehara N, Manabe A. Kawasaki disease shock syndrome: case report and cytokine profiling. Pediatr Int. 2019;61:620-2.

5. Dufort EM, Koumans EH, Chow EJ, Rosenthal EM, Muse A, Rowlands J, et al. Multisystem inflammatory syndrome in children in New York State. N Engl J Med. 2020;383:347-58.

6. Feldstein LR, Rose EB, Horwitz SM, Collins JP, Newhams $\mathrm{MM}$, Son MBF, et al. Multisystem inflammatory syndrome in U.S. children and adolescents. N Engl J Med. 2020;383:334-46.

7. IDWR Surveillance Data Table 2020 week 34. https://www.niid. go.jp/niid/ja/data.html. Accessed 5 Sept 2020.

Publisher's Note Springer Nature remains neutral with regard to jurisdictional claims in published maps and institutional affiliations. 